OPEN ACCESS

Edited by: Jianming $L i$,

Soochow University, China

Reviewed by: Jun Zhou,

Southern Medical University, China Antonio leni,

University of Messina, Italy

*Correspondence:

Ben-Chun Jiang jiangbenchun@yeah.net

Specialty section: This article was submitted to Gastrointestinal Cancers, a section of the journal

Frontiers in Oncology

Received: 07 September 2020 Accepted: 12 November 2020 Published: 23 December 2020

Citation:

Ji C, Sun L-S, Xing F, Niu N, Gao H-L, Dai J-W, Zhou N and Jiang B-C (2020)

HTRA3 Is a Prognostic Biomarker and Associated With Immune Infiltrates in Gastric Cancer.

Front. Oncol. 10:603480. doi: 10.3389/fonc. 2020.603480

\section{HTRA3 Is a Prognostic Biomarker and Associated With Immune Infiltrates in Gastric Cancer}

\author{
Ce $\mathrm{Ji}^{1,2}$, Li-Sha Sun ${ }^{2,3}$, Fei Xing ${ }^{2,3}, \mathrm{Nan} \mathrm{Niu}^{2,4}$, Hong-Li Gao ${ }^{2,3}$, Jing-Wei Dai ${ }^{5}$, Nan Zhou ${ }^{1}$ \\ and Ben-Chun Jiang ${ }^{1 *}$ \\ ${ }^{1}$ Department of General Surgery, Shengjing Hospital of China Medical University, Shenyang, China, ${ }^{2}$ Tumor Stem Cell and \\ Transforming Medicine Laboratory, Shengjing Hospital of China Medical University, Benxi, China, ${ }^{3}$ Department of Breast \\ Surgery, Shengjing Hospital of China Medical University, Shenyang, China, ${ }^{4}$ Department of Oncology, Shengjing Hospital of \\ China Medical University, Shenyang, China, ${ }^{5}$ Department of Neurosurgery, Shengjing Hospital of China Medical University, \\ Shenyang, China
}

HtrA serine peptidase 3 (HTRA3) participates in multiple signal pathways and plays an important regulatory role in various malignancies; however, its role on prognosis and immune infiltrates in gastric cancer (GC) remains unclear. The study investigated HTRA3 expression in tumor tissues and its association with immune infiltrates, and determined its prognostic roles in GC patients. Patients with GC were collected from the cancer genome atlas (TCGA). We compared the expression of HTRA3 in GC and normal gastric mucosa tissues with Wilcoxon rank sum test. And logistic regression was used to evaluate the relationship between HTRA3 and clinicopathological characters. Gene ontology (GO) term analysis, Gene set enrichment analysis (GSEA), and single-sample Gene Set Enrichment Analysis (SSGSEA) was conducted to explain the enrichmental pathways and functions and quantify the extent of immune cells infiltration for HTRA3. Kaplan-Meier analysis and Cox regression were performed to evaluate the correlation between HTRA3 and survival rates. A nomogram, based on Cox multivariate analysis, was used to predict the impact of HTRA3 on prognosis. High HTRA3 expression was significantly correlated with tumor histological type, histological grade, clinical stage, T stage, and TP53 status $(P<0.05)$. HTRA3-high GC patients had a lower 10-year progression-free interval [PFI; hazard ratio $(\mathrm{HR})$ : 1.46; 95\% confidence interval $(\mathrm{Cl})$ : 1.02-2.08; $P=0.038]$, disease-specific survival (DSS; HR: 1.65; Cl: 1.08-2.52; $P=0.021$ ) and overall survival (OS; HR: 1.59; Cl: $1.14-$ 2.22; $P=0.006)$. Multivariate survival analysis showed that HTRA3 was an independent prognostic marker for PFI (HR: 1.456; Cl: 1.021-2.078; $P=0.038)$, DSS (HR: 1.650; Cl: 1.079-2.522; $P=0.021$ ) and OS [hazard ratio $(\mathrm{HR}): 1.590 ; 95 \%$ confidence interval $(\mathrm{Cl})$ :1.140-2.219; $P=0.006$ ]. The $C$-indexes and calibration plots of the nomogram based on multivariate analysis indicated an effective predictive performance for GC patients. GSEA showed that High HTRA3 expression may activate NF-kB pathway, YAP1/ WWTR1/TAZ pathway, and TGF $\beta$ pathway. There was a negative correlation between the HTRA3 expression and the abundances of adaptive immunocytes (T helper cell 17 cells) and a positive correlation with abundances of innate immunocytes (natural killer cells, 
macrophages etc.). HTRA3 plays a vital role in GC progression and prognosis and could be a moderate biomarker for prediction for survival after gastrectomy.

Keywords: HTRA3, bioinformatics, biomarker, gastric cancer, immune infiltration, prognosis

\section{INTRODUCTION}

Gastric cancer (GC) is one of the most lethal carcinomas worldwide, and almost half of all GC cases are diagnosed in East Asia (1). Despite the developments in multimodal therapy strategies, such as surgery, chemotherapy, radiotherapy and immunotherapy, the five-year survival rates for the patients with stage II and stage IIIA GC were about 34 and 20\%, respectively (2). GC is a heterogeneous malignancy $(2,3)$ and the existing microsatellite instability, HER2 mutation, and amplification cannot completely explain the different prognosis or therapeutic response of GC $(4,5)$. And the current biomarkers for the prognosis of GC are not suitable for clinical needs. Numerous studies currently on molecular targeted therapy and associated molecular pathways referred to the gastric tumorigenesis have illuminated the pathogenesis of GC and helped ameliorate the prognosis of patients with GC (6). For example, Trastuzumab can extend the survival time of the patients with HER2-positive GC (7). Therefore, there is an urgent necessity for the elucidation of the identification of novel biomarkers for carcinoma diagnosis and therapeutic targets in GC.

HtrA Serine Peptidase 3 (HTRA3), which was first reported in 2003 , is a protein encoding gene located on chromosome 4p16.1 (8). HTRA3 protein (expressed in many cell types and organs) has two isomers, which both represent active serine proteases which cleave beta-casein/CSN2 as well as several extracellular matrix (ECM) proteoglycans (9). Previous studies indicated that HTRA3 inhibits signaling mediated by TGF-beta family proteins possibly indirectly by degradation of these ECM proteoglycans (10).

HTRA3 was first shown to be associated with cancer in a report demonstrating that it may act as a tumor suppressor, which is a proapoptotic protease that promotes drug-induced cytotoxic effects in lung cancer cells (11). Overexpressed HTRA3 inhibits the carcinogenic role of TGF $\beta 1$ and thus inhibits metastasis in the early stages of non-small cell lung cancer (10). HTRA3 expression was negatively correlated with lymph node metastasis in breast cancer, but not with positive or negative expression of ER and PR (12). Recent studies illuminated it represents as a tumor promoter. The expression of HTRA3 in the peritumor stroma of patients with stage II colorectal cancer is associated with high-grade tumor budding, which may be a new marker of poor prognosis (13). HTRA3 may be related to the acquisition of invasive phenotype of and may be a potential prognostic indicator of oral cancer (14). These findings suggest that HTRA3 has multifaceted functional roles in various malignancies. Moreover, the underlying functions and mechanisms of HTRA3 in tumor progression and tumor immunology are still unclear.

In this study, we aimed to systematically analyze the significance of HTRA3 in GC using RNA sequencing data retrieved from TCGA database, along with bioinformatics and statistical methods including differentially expressed genes (DEG) analysis, gene ontology (GO) term analysis, Kyoto Encyclopedia of Genes and Genomes (KEGG) pathway analysis, gene set enrichment analysis (GSEA), single-sample gene set enrichment analysis (ssGSEA), Kaplan-Meier survival analysis, and logistic \& Cox regression analysis. Moreover, we further developed a nomogram to predict the patients' prognosis.

\section{MATERIAL AND METHODS}

\section{Data Source and Preprocessing}

Gene expression data with clinical information from STAD projects (included 32 normal and 375 tumor tissues, Workflow Type: HTSeq-FPKM) were collected from TCGA. The exclusion criteria were normal STAD samples and an overall survival less than 30 days. Next, level 3 HTSeq-FPKM data were transformed into TPM (transcripts per million reads), and the TPM data of 375 GC patients were used for further analyses. Unavailable or unknown clinical features were regarded as missing values. The data are summarized in Supplemental Table 1.

\section{HTRA3 Differential Expression in GC Tissues in the TCGA Database}

Boxplots and scatter plots, using disease state (tumor or normal) as the variable, were generated to calculate differential expression of HTRA3. The diagnostic performance of HTRA3 was estimated using receiver operating characteristic (ROC) curves. Statistical ranking for HTRA3 expression above or below the median value was defined as HTRA3-high or HTRA3low, respectively.

\section{Experimental Verification of HTRA3 Differential Expression in GC Tissues and Cell Lines With Quantitative Polymerase Chain Reaction (qPCR) and Western Blotting}

A total of 44 pairs of human GC samples and non-malignant gastric tissues which at least $5 \mathrm{~cm}$ away from the tumor samples were collected from patients who underwent a gastrectomy at the Department of Surgery, Shengjing Hospital, China Medical University between January 2019 and December 2019. All GC cases were pathologically confirmed. Five human GC cell lines (SGC7901, BGC823, MGC803, HGC27, and MKN45) and one immortalized normal gastric cell line (GES1) were obtained from the Institute of Biochemistry and Cell Biology, Chinese Academy of Sciences (Shanghai, P.R. China). Total RNA and proteins were obtained from these specimens. 
The expression of HTRA3 mRNA was detected using qPCR with the following program: $95^{\circ} \mathrm{C}$ for $30 \mathrm{~s}, 40$ cycles of $95^{\circ} \mathrm{C}$ for $5 \mathrm{~s}$, and $60^{\circ} \mathrm{C}$ for $30 \mathrm{~s}$. The reaction mixture contained $10 \mu \mathrm{l}$ SYBR Green (Takara, Dalian, China), $0.4 \mu \mathrm{l}$ each primer, $2 \mu \mathrm{l}$ cDNA, and $7.2 \mu$ diethylpyrocarbonate (DEPC)-treated water. Real-time PCR was performed according to the protocol of SYBR Premix Ex TaqTMII kit. The primers used were as follows: Sense: 5' -CTGAGACACCCGCTGTTTG- 3' and antisense: 5' -CCATTCTGTAGCTGCACCTT- 3' for HTRA3; and sense: 5' -TGACTTCAACAGCGACACCCA-3' and antisense: 5'-CACCCTGTTGCTGTAGCCAAA-3' for GADPH. Gene expression levels were calculated relative to the housekeeping gene GAPDH.

Each sample $(60 \mu \mathrm{g})$ was electrophoresed in $10 \%$ polyacrylamide gel and transferred to a polyvinylidene difluoride membrane (Millipore, Bedford, MA, USA) using a BG-blotMiMi transfer machine (Baygene, Beijing, China). After blocking with $5 \%$ nonfat milk for $2 \mathrm{~h}$ at room temperature (about 20 to $25^{\circ} \mathrm{C}$.), overnight incubation with primary antibodies for HTRA3 (1:500 dilution; Abcom) or GAPDH (1:2,000 dilution; Santa Cruz) was performed at $4^{\circ} \mathrm{C}$. The next day, after incubation with secondary antibodies (1:5000; Santa Cruz) at $37^{\circ} \mathrm{C}$ for $2 \mathrm{~h}$, and after washing, the immunoreactive protein bands were visualized using an electrochemiluminescence (ECL) detection kit (Thermol Biotech, Rockford, IL, USA). The ratio between the optical density of the protein and GAPDH was calculated as the relative content of the protein detected. Each experiment was repeated three times.

\section{Analysis of DEGs Between HTRA3-High and -Low Expression GC Groups}

DEGs between HTRA3-high and HTRA3-low patients from TCGA datasets were identified by the unpaired Student's t-test, within the DESeq2 (3.8) package (15). Genes with the adjusted $P$ value $<0.05$ and the absolute FC larger than 1.5 were considered to be statistically significant. All the DEGs were presented in a heat map and volcano plots.

\section{Functional Enrichment and Analysis of Immune Cell Infiltration}

In this study, Metascape (http://metasape.org) (16) was used as a tool to analyze the enrichment of HTRA3 related DEGs by process and pathway. The threshold conditions included: $P<0.01$, a minimum count of 3 , and the enrichment factor $>1.5$ to obtain significant statistical differences.

GSEA starts with the HTRA3 differentially expressed matrix and analyzes the differences in signal pathways between the HTRA3-high and -low groups to predict the HTRA3-related phenotypes and signal pathways. A permutation test with 1,000 times was used to identify the significantly changed pathways. Adjusted $\mathrm{P}<0.01$ and FDR $<0.25$ were identified as significant related genes. Statistical analysis and graphical plotting were conducted using R package clusterProfiler (3.8.0) (17). To construct the protein-protein interaction (PPI) network, the DEGs were input into STRING database (18). And PPI pairs with an interaction score $>0.95$ were chosen to build the PPI network.

The relative tumor infiltration levels of 24 immune cell types were quantified by ssGSEA to interrogate expression levels of genes in published signature gene lists (19). The signatures we used included a diverse set of adaptive and innate immune cell types and comprised 509 genes in total. To explore the correlation between HTRA3 and the infiltration levels of immune cells and the association of infiltration of immune cells with the different expression groups of HTRA3, Wilcoxon rank sum test, and Spearman correlation were adopted.

\section{Clinical Statistical Analysis on Prognosis, Model Construction, and Evaluation}

All statistical analyses were performed in $\mathrm{R}$ package (V3.6.2). The relationship between clinical pathologic features and HTRA3 were analyzed with the Wilcoxon signed-rank sum test and logistic regression. Clinicopathological characteristics associated with the 10-year overall survival (OS), progressionfree interval (PFI), and disease-specific survival (DSS) in TCGA patients using Cox regression and the Kaplan-Meier method. Multivariate Cox analysis was used to compare the influence of HTRA3 expression on survival along with other clinical characteristics (stage, myometrial invasion, lymph node status, distant metastasis status, histological grade and subtype). The cut-off value of HTRA3 expression was determined by its median value. P-values less than 0.05 were considered significant in all tests. The difference of 10-year OS, PFI, and DSS between HTRA3-high and -low group was calculated by the KaplanMeier method with a two-sided log-rank test.

Based on Cox regression models, the independent prognostic factors obtained from multivariate analysis were used to establish nomograms, individualizing the predicted survival probability for 1-, 3-, and 5-year. The RMS package (Version: 5.1-4; https:// cran.r-project.org/web/packages/rms/index.html) was employed to generate nomograms that included significant clinical characteristics and calibration plots. The calibration curves were graphically assessed by mapping the nomogram-predicted probabilities against the observed occurrences, and the $45^{\circ}$ line represented the best predictive values. A concordance index (Cindex) was used to determine the discrimination of the nomogram, and it was calculated by a bootstrap approach with 1,000 resamples. The predictive accuracies of the nomogram and separate prognostic factors were compared using the C-index. All statistical tests were two tailed with a statistical significance level set at 0.05 in this study.

\section{RESULTS}

\section{Abnormally High Expression of HTRA3 in GC}

Firstly, the pan-cancer analyses were performed to compare the expression of HTRA3 in the tumor samples of GTEx combined with TCGA and the corresponding normal samples of TCGA by 
Wilcoxon rank sum test. HTRA3 was significantly expressed in adrenocortical carcinoma (ACC), bladder urothelial carcinoma (BLCA), breast infiltrating carcinoma (BRCA), cervical squamous cell carcinoma and adenocarcinoma (CESC), cholangiocarcinoma $(\mathrm{CHOL})$, diffuse large $\mathrm{B}$ cell lymphoma (DLBCL), pleomorphic glioma (GBM), head and neck squamous cell carcinoma (HNSC), renal chromophobe cell carcinoma $(\mathrm{KICH})$, renal clear cell carcinoma (KIRC), renal papillary cell carcinoma (KIRP), acute myeloid leukemia (LAML), brain low grade glioma (LGG), lung adenocarcinoma (LUAD), ovarian serous cystadenocarcinoma (OV), Pancreatic cancer (PAAD), prostate cancer (PRAD), skin melanoma (SKCM), gastric cancer (STAD), thyroid cancer (THCA), thymic cancer (THYM), endometrial cancer (UCEC), uterine sarcoma (UCS) $(P<0.05)$ (Figure 1A). Secondly, we compared the expression of HTRA3 in 32 paracancerous samples and 375 GC samples in TCGA STAD dataset. The expression of HTRA3 was significantly high in GC samples $(P=0.002)$ (Figure 1B; Supplementary Table 1). However, there was no significant difference in the expression of HTRA3 in 27 GC samples and matched paracancerous samples $(\mathrm{p}=0.117)$ (Figure $1 \mathrm{C})$, that may be due to the small number of paired samples in TCGA database. Therefore, we expanded the number of paired samples to verify mRNA and protein differential expression of HTRA3 in gastric cancer tissues and cell lines. We verified that HTRA3 is highly expressed in GC tissues by QPCR $(P=0.0035)$ and WB analysis $(P=0.0013)$ (Supplementary Figure 1A) on 44 paired GC samples and paracancerous samples. Among the GC cells, HTRA3 mRNA and protein expression was found at a comparatively higher level in MGC803, HGC27, and MKN45 cells and was lowest in SGC7901 (Supplementary Figure 1B).

ROC was used to analyze the distinguishing efficacy of HTRA3 between GC tissues and normal gastric mucosa tissue. The area under the curve (AUC) of HTRA3 is 0.710 , suggesting

A

-TCGA+GTEx_Normal - TCGA_Tumor

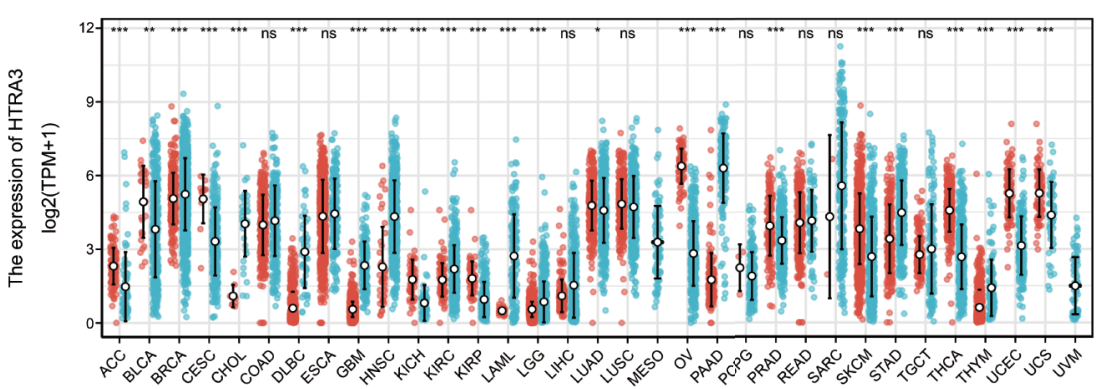

B

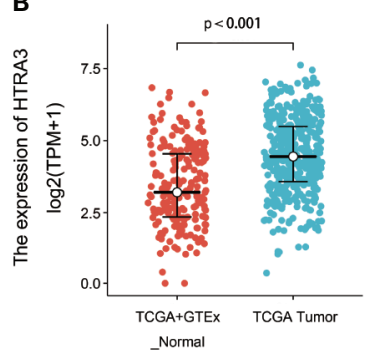

E

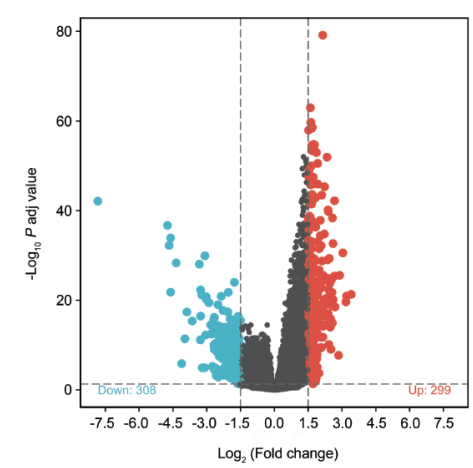

D
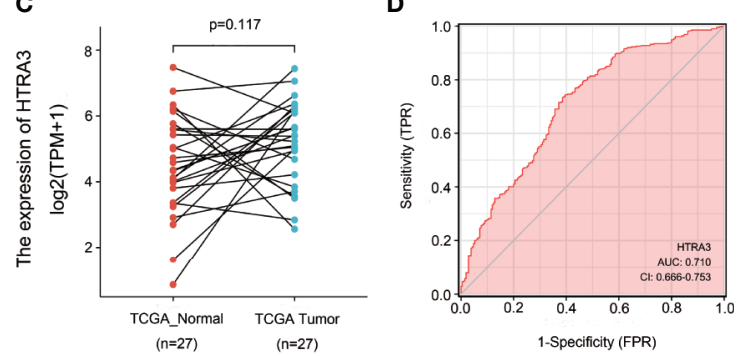

$\mathbf{F}$

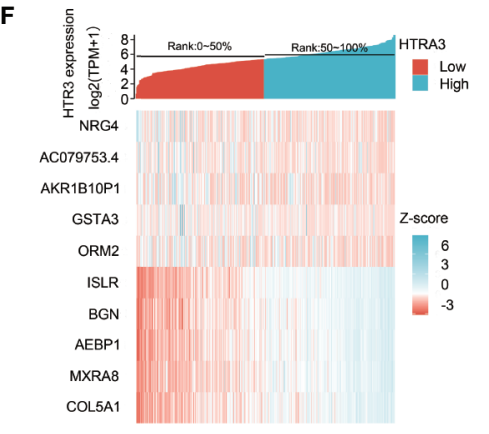

FIGURE 1 | Differential expression levels of HTRA3 in different malignancies and HTRA3-related differentially expressed genes (DEGs). (A) Increased or decreased HTRA3 of different cancers compared with normal tissues in the TCGA and GTEx database. (B, C) Differential expression levels of HTRA3 in GC. (D) A ROC curve to test the value of HTRA3 to identify GC tissues was created. (E, F) Volcano plots of the DEGs and heat map showing the top 10 DEGs. 
that HTRA3 may be a potentially moderate identification molecule for GC tissues (Figure 1D).

\section{Identification of DEGs in GC}

We compared 187 GC HTRA3-high samples with 188 HTRA3low controls. A total of 607 DEGs, covering 299 upregulated GENEs and 308 downregulated GENEs, were identified to be statistically significant between the two cohorts (adjusted p-value < 0.05, $\mid$ Log2-fold change $>1.5$ ) (Figure 1E; Supplementary Table 2). Then, DEGs in HTSeq-Counts were further analyzed by DESeq2 package. Relative expression values of the top 10 DEGs between the two cohorts were showed in Figure 1F.

\section{Functional Enrichment and Analyses of HTRA3 Related Genes in GC}

In order to predict the functional enrichment information of HTRA3 interactive genes, we used Metascape for GO enrichment analysis, which showed that HTRA3-related genes were involved in many biological processes (BPs), cellular compositions (CCs), and molecular functions (MFs), including extracellular matrix organization, extracellular structure organization, transmembrane receptor protein serine/threonine kinase signaling pathway. Moreover, regulation of transmembrane receptor protein serine/ threonine kinase signaling pathway, extracellular matrix disassembly, regulation of extrinsic apoptotic signaling pathway via death domain receptors, and positive regulation of cell-substrate adhesion were also involved in the regulation of HTRA3 interactive genes (Figure 2A; Supplementary Table 3).

\section{Protein-Protein Interaction (PPI) Network Analysis}

To obtain the interactions between the 607 DEGs in the GC group, a PPI network was constructed using the STRING database and the interaction threshold is set at 0.90. A total of 319 proteins and 1,425 edges were selected and 4 hub gene clusters were selected from PPI network with scores $\geq 6,600$ (Supplementary Figures 2A-E). Additionally, top 10 hub genes included IVL, TGM1, SPRR3, SPRR2A, SPRR2E, SPRR2D, LCE3E, SPRR2B, LCE3D, and SPRR2G.

\section{GSEA Identifies HTRA3-Related Signaling Pathways}

To identify HTRA3-related signaling pathways in GC, GSEA between HTRA3-high and -low expression data sets was conducted to reveal significant differences (adjusted $P<0.05$, FDR q value < 0.25) in enrichment of MSigDB Collection (c2.cp.biocarta and hall. v6.1 symbols). The most significantly enriched signaling pathways based on their normalized enrichment score (HES) were selected. Moreover, the differentially enriched pathways in HTRA3 low expression phenotype include NF- $\mathrm{KB}$ signaling pathway, YAP1/WWTR1/ TAZ signaling pathway, TGF $\beta$ pathway, RAS/CA2P/NMDA pathway, RHO/CIT pathway, and RAC1 signaling pathway (Figures 2B-G; Supplementary Table 5).

\section{The Correlation Between HTRA3 Expression and Immune Infiltration}

The correlation between the expression level (TPM) of HTRA3 and immune cell infiltration level quantified by ssGSEA was analyzed by spearman correlation. The expression of HTRA3 was negatively correlated with the abundance of acquired immunocytes [helper T17 (Th17) cells, T helper cells, T central memory cells, etc.], and positively correlated with the abundance of innate immunocytes [natural killer (NK) cells, tumorassociated macrophages (TAMs), immature dendritic cells, etc.] (Figures 3A-G, $P<0.001$ ).

\section{Association With HTRA3 Expression and Clinicopathological Variables}

To clarify the role and significance of HTRA3 expression, a total of 375 GC samples with HTRA3 expression data with all patients' characteristics were analyzed from TCGA. The cohort included 241 men and 134 women with an average age of 61.1 years (range 51-69 years). As shown in Figures 4A-E and Table 1, overexpressed HTRA3 was significantly correlated with tumor histological type (mucinous type vs. tubular type, $P=0.003$ ), histological grade (grade 3 vs. grade 1 and $2, P<$ 0.001 ), clinical stage (stage IV vs. stage I, $P<0.001$ ), T stage (T4 vs. T1, $P<0.001$ ), and TP53 status (wild type vs. mutational type, $P=0.014)$. HTRA3 expression has no relation with other clinicopathological characteristics (Figures 4F-I). The univariate analysis with Logistic regression illuminated HTRA3 expression as a categorical dependent variable was associated with poor prognostic clinicopathological characteristics (Table 2). Increased HTTA3 expression in GC is positively associated with $\mathrm{T}$ stage $(\mathrm{OR}=2.39$ for $\mathrm{T} 3$ and $\mathrm{T} 4$ vs. $\mathrm{T} 1$ and $\mathrm{T} 2)$, histological grade $(\mathrm{OR}=2.14$ for $\mathrm{G} 3$ vs. $\mathrm{G} 1$ and $\mathrm{G} 2)$; meanwhile negatively associated with TP53 status $(\mathrm{OR}=0.59$ for mutation vs. wild type) significantly (all $P<0.05$ ). These results suggested that GCs with high HTRA3 expression were prone to progress to a more advanced stage and less susceptible to TP53 mutations than those with low HTRA3 expression.

\section{High HTRA3 Expression Was Closely Associated With Poor Prognosis of Patients With GC}

The 10-year OS rates were significantly higher among patients with low HTRA3 expression than those with high HTRA3 expression (37.6 vs. 24.7\%; $P=0.006$; Figure 5A). Similarly, the 10-year PFI rates and DSS in the HTRA3-low group were significantly higher than those in the HTRA3-high group (46.9 vs. $36.7 \%$; $P=0.038 ; 57.3$ vs. $38.2 \% ; P=0.021$; Figures $5 B, \mathbf{C}$ ).

Next, we conducted subgroup survival analyses of OS, DSS, and PFI, which showed that the prognosis of patients with HTRA3-high was poor in stage III-IV, stage III, T4, N2\&N3, and M0 subgroups of OS and M0 subgroup of DSS (Figures 5D-I). Furthermore, it should be noted that the GC patients with HTRA3-high in M0 subgroup had worse OS and DSS (33.2 vs. $22.7 \%$; $P=0.019 ; 54.3$ vs. $37.2 \% ; P=0.029)$, indicating HTRA3 had a greater prognostic role in GC patients without distant metastasis. However, there 

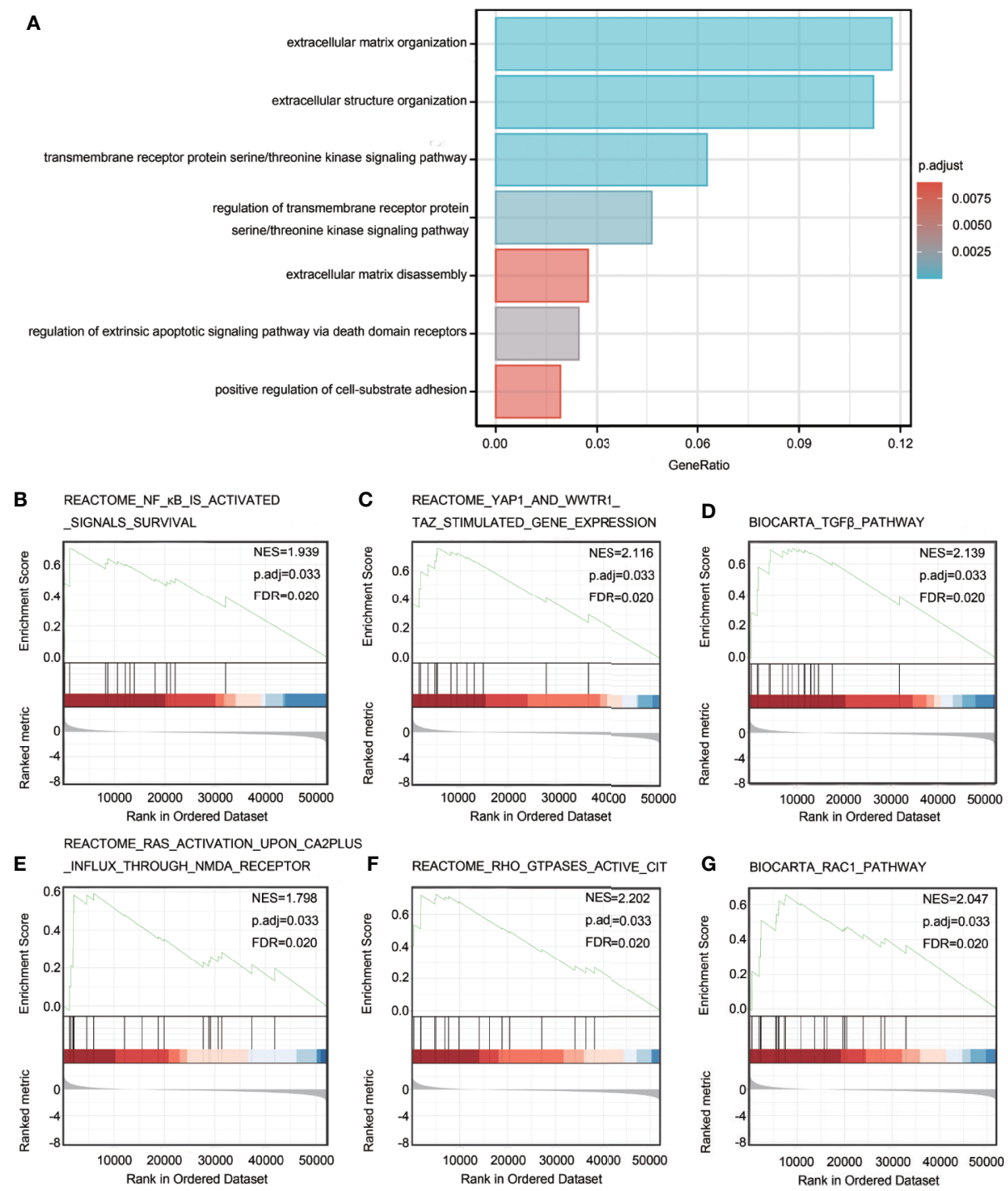

FIGURE 2 | Significantly enriched GO annotations of HTRA3 related genes in GC. (A) Top 7 of biological process enrichment related to HTRA3 related genes with bar graph. (B-G) Enrichment plots from the gene set enrichment analysis (GSEA). Several pathways and biological processes were differentially enriched in HTRA3related GC, including activated NF-KB signals survival, YAP1 and WWTR1-TAZ stimulated gene expression, TGF $\beta$ pathway, RAS activation upon $\mathrm{Ca}^{2+}$ influx through NMDA receptor, active CIT activated by RHO GTPases, and RAC1 signaling pathway. NES, normalized enrichment score; p.adj, adjusted P value; FDR, false discovery rate.

was no significant difference in survival among each subgroup of PFI.

A univariate logistic regression indicated that higher HTRA3 expression was associated with a short OS [hazard ratio (HR): 1.590; 95\% confidence interval (CI):1.140-2.219; $P=0.006]$ and poor PFI (HR: 1.456; CI: 1.021-2.078; $P=0.038$ ) as well as DSS (HR: 1.650; CI: $1.079-2.522 ; P=0.021$ ) (Table 3 and Supplemental Tables 6, 7). To further seek factors associated with survival, a multivariate Cox regression analysis was performed with pathologic stage, $\mathrm{T}$ stage, $\mathrm{N}$ stage, $\mathrm{M}$ stage, Histological grade, age, primary therapy outcome, and residual tumor status. High HTRA3 expression was still an independent factor associated with poor OS (HR: 2.315; CI: 1.447-3.703; $P<$
0.001) (Table 3). However, HTRA3 expression levels showed no association with poor DSS (HR: 1.405; CI: $0.824-2.395 ; P=$ $0.211)$ (Supplemental Table 6) and short PFI (HR: 1.116; CI: $0.656-1.898 ; P=0.686$ ) (Supplemental Table 7) in patients with GC.

Based on the independent adverse prognostic factors chosen by multivariate Cox analysis, we studied the effect of HTRA3 expression on prognosis (OS, PFI, and DSS) in different subgroups. The HTRA3-high GC patients had shorter OS time in the T4 subgroup (HR: 2.130; CI: 1.106-4.104; $P=0.024$ ), N2\&3 subgroup (HR: 1.979; CI: $1.214-3.227 ; P=0.006$ ), M0 subgroup (HR: 1.536; CI: 1.075-2.196; $P=0.019$ ) and pathologic stage III subgroup (HR: 2.244; CI: $1.040-4.838 ; P=0.039$ ) and 
A

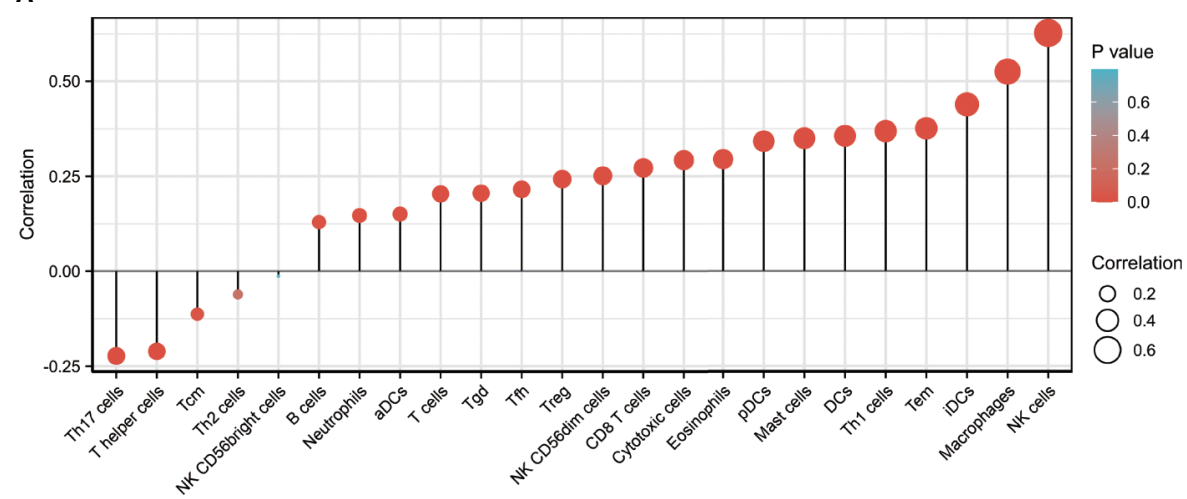

B

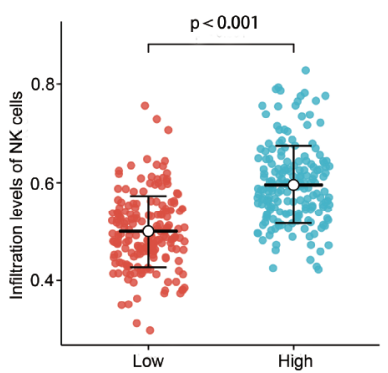

E

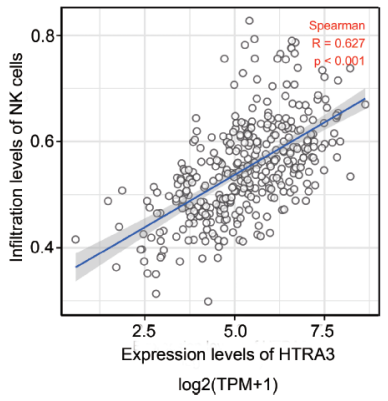

C

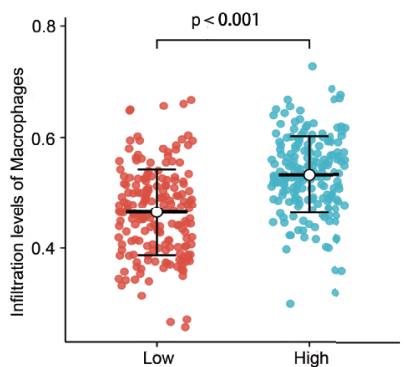

$\mathbf{F}$

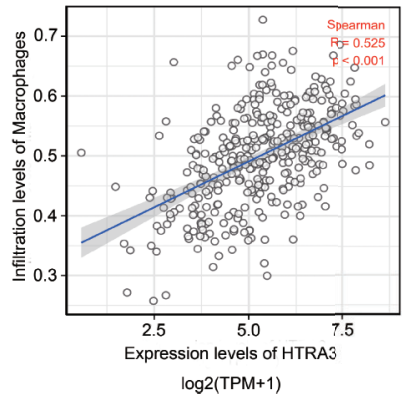

D

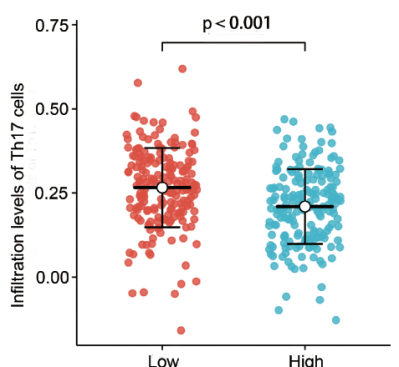

G

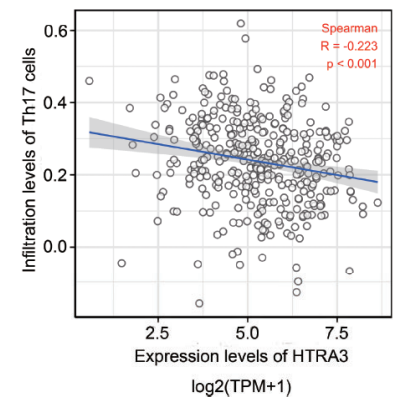

FIGURE 3 | The expression level of HTRA3 was associated with the immune infiltration in the tumor microenvironment. (A) Correlation between the relative abundances of 24 immune cells and HTRA3 expression level. The size of dots shows the absolute value of Spearman R. (B-G) Scatter plots and correlation diagrams showing the difference of NK cells, Macrophages, and Th17 cells infiltration level between HTRA3-high and -low groups.

stage III \& IV subgroup (HR: 1.546; CI: 1.013-2.357; $P=0.043$ ) (Table 4). Similarly, the DSS prognostic analyses showed that the HTRA3-high GC patients had a shorter survival time in M0 subgroup (HR: 1.665; CI: 1.055-2.627; $P=0.029$ ) (Supplemental Table 8). However, the PFI prognostic analyses did not find that the difference in HTRA3 expression had a significant effect on the prognosis in any subgroup (Supplemental Table 9).

\section{Construction and Validation of a Nomogram Based on the HTRA3}

To provide a quantitative approach predicting the prognosis of GC patients, HTRA3 and independent clinical risk factors were used to construct a nomogram (Figure 6A). In the nomogram based on multivariate Cox analysis, a point scale was used to assign points to these variables. The sum of points assigned to each variable was readjusted to a range from 1 to 100 . The points of the variables were accumulated and recorded as the total scores. The probability of survival in GC patients at 1,3 , and 5 years was determined by drawing a vertical line directly down from the total point axis to the outcome axis. For instance, a GC patient with high HTRA3 risk (45 points), lymph node metastasis ( 42.5 points), and poor therapy outcome (37 points) received a total point score of 124.5. The probabilities of 1-, 3-, 5year survival were about $87.5,57$, and $38 \%$ (Figure 6A).

We also analyzed the prediction efficiency of the nomogram, and the result illuminated that the $\mathrm{C}$-index of the model was 0.743(CI: 0.720-0.766), which suggested that the prediction efficiency of this model is moderately accurate. The biascorrected line in the calibration plot was utilized to be close to the ideal curve (the 45-degree line), which showed a fine 

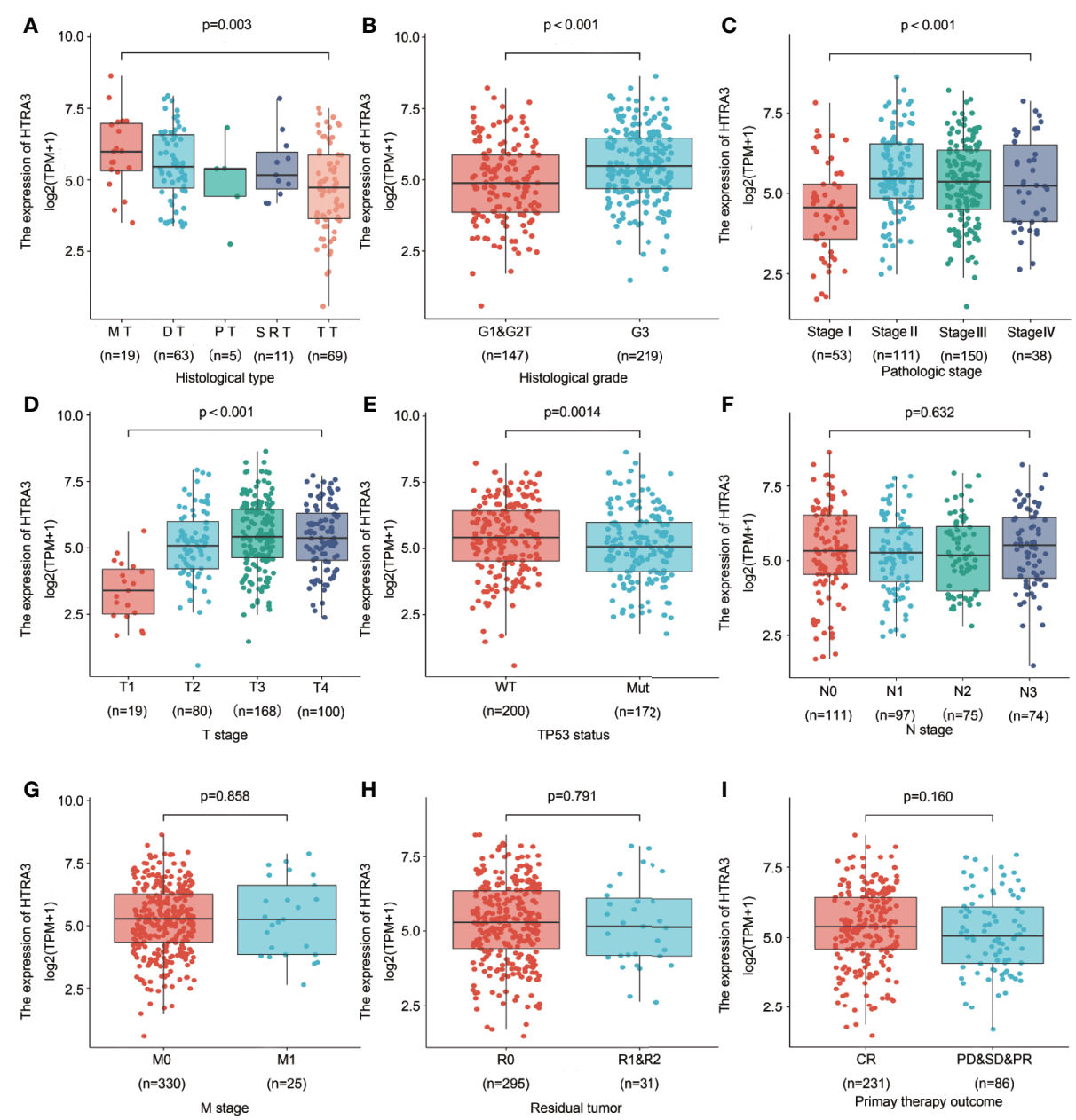

FIGURE 4 | Association with HTRA3 expression and clinicopathological characteristics, including (A) histological type, (B) histological grade, (C) pathologic stage, (D) T stage (E) TP53 status, (F) N stage, (G) M stage, (H) residual tumor, and (I) primary therapy outcome in GC patients in TCGA cohort. TCGA, The Cancer Genome Atlas; GC, gastric cancer; MT, mucinous type; DT, diffuse type; PT, papillary type; SRT, signet ring type; T, tubular type.

agreement between the prediction and the observation (Figure 6B). Moreover, the nomogram performance of HTRA3 (Cindex: 0.743$)$ was better than the performance of $\mathrm{T}$ stage (C-index: 0.698), $M$ stage (C-index: 0.677), pathological stage (C-index: 0.722). These results suggested that the nomogram was a better model for predicting short- or long-term survival in patients with GC than individual prognostic factors.

\section{DISCUSSION}

To our knowledge, the expression of HTRA3 and its potential prognostic impact on GC has not been explored. Hence, the potential role of HTRA3 in GC was the focus of the present study. In the present study, bioinformatics analysis using highthroughput RNA-sequence data from TCGA database demonstrated that there were evident individual variation and heterogeneity in these RNA transcripts, and HTRA3 might be a potential moderate marker for GC tissues. Increased expression of HTRA3 in GC was associated with fewer TP53 mutations, advanced clinical pathologic characteristics, shorter survival time, and poor prognosis.

Human HTRA3 is a trimer multitasking serine protease related to cell function and pathogenicity. It may serve as a potential therapeutic target (9). HTRA3 dissolves XIAP and $\mathrm{BAX}$, therefore promoting apoptosis. Additionally, it is associated with epithelial-mesenchymal transformation (EMT) (11). HTRA3 may inhibit the signal transduction of TGF- $\beta$ family members and prohibit cell migration through MEK/ ERK pathway (20). HTRA3 may promote the instability of actin and vimentin cytoskeleton and affect the dynamics of cytoskeleton (21).

In some malignancies, HTRA3 has anti-tumor effect. Previous studies have elucidated the low expression of HTRA3 in endometrial carcinoma and ovarian cancer had anti-tumor effect $(22,23)$. The ectopic expression of HTRA3 leaded to the decrease of cell proliferation and the increase of the expression of apoptotic protein Bax, suggesting that HTRA3 has anti-tumor 
TABLE 1 | The association between HTRA3 expression and clinicopathological variables. Abbreviations: ${ }^{a}$ statistically significant ${ }^{\mathrm{b}}$ Exact $\chi^{2}$ test.

\begin{tabular}{|c|c|c|c|c|c|}
\hline Characters & level & Low expression of HTRA3 & High expression of HTRA3 & $\mathrm{p}$ & test \\
\hline \multirow[t]{2}{*}{ T stage (\%) } & T1 & $18(9.8 \%)$ & $1(0.5 \%)$ & $<0.001$ & exact \\
\hline & T4 & $47(25.5 \%)$ & $53(29.0 \%)$ & & \\
\hline \multirow[t]{2}{*}{ N stage (\%) } & NO & $53(29.8 \%)$ & $58(32.4 \%)$ & 0.589 & exact \\
\hline & $\mathrm{N} 1$ & $52(29.2 \%)$ & $45(25.1 \%)$ & & \\
\hline \multirow[t]{2}{*}{ M stage (\%) } & MO & $167(92.8 \%)$ & $163(93.1 \%)$ & 1.000 & exact \\
\hline & M1 & $13(7.2 \%)$ & $12(6.9 \%)$ & & \\
\hline \multirow[t]{4}{*}{ Pathologic stage (\%) } & Stage I & $38(21.7 \%)$ & $15(8.5 \%)$ & 0.002 & exact \\
\hline & Stage II & $46(26.3 \%)$ & $65(36.7 \%)$ & & \\
\hline & Stage III & $70(40.0 \%)$ & $80(45.2 \%)$ & & \\
\hline & Stage IV & $21(12.0 \%)$ & $17(9.6 \%)$ & & \\
\hline \multirow{2}{*}{ Primary therapy outcome (\%) } & $\mathrm{PR}$ & $2(1.3 \%)$ & $2(1.2 \%)$ & & \\
\hline & SD & $9(5.8 \%)$ & $8(4.9 \%)$ & & \\
\hline \multirow[t]{2}{*}{ Gender (\%) } & Female & 65(34.6\%) & $69(36.9 \%)$ & 0.718 & \\
\hline & Male & $123(65.4 \%)$ & $118(63.1 \%)$ & & \\
\hline \multirow[t]{3}{*}{ Race (\%) } & Asian & $41(26.8 \%)$ & $33(19.4 \%)$ & $<0.001$ & \\
\hline & Black or African American & $11(7.2 \%)$ & $0(0.0 \%)$ & & \\
\hline & White & $101(66.0 \%)$ & $137(80.6 \%)$ & & \\
\hline \multirow[t]{2}{*}{ Age (\%) } & $<=65$ & $78(42.2 \%)$ & $86(46.2 \%)$ & 0.493 & \\
\hline & $>65$ & $107(57.8 \%)$ & $100(53.8 \%)$ & & \\
\hline \multirow[t]{4}{*}{ Histological type (\%) } & Diffuse Type & $28(15.0 \%)$ & $35(18.7 \%)$ & 0.031 & exact \\
\hline & Mucinous Type & $5(2.7 \%)$ & $14(7.5 \%)$ & & \\
\hline & Not Otherwise Specified & $101(54.0 \%)$ & $106(56.7 \%)$ & & \\
\hline & Papillary Type & $2(1.1 \%)$ & $3(1.6 \%)$ & & \\
\hline \multirow{4}{*}{ Anatomic neoplasm subdivision (\%) } & Cardia/Proximal & $25(13.9 \%)$ & $23(12.7 \%)$ & & \\
\hline & Fundus/Body & $59(32.8 \%)$ & $71(39.2 \%)$ & & \\
\hline & Gastroesophageal Junction & $27(15.0 \%)$ & $14(7.7 \%)$ & & \\
\hline & Other & $4(2.2 \%)$ & $0(0.0 \%)$ & & \\
\hline \multirow[t]{2}{*}{ Reflux history (\%) } & No & $90(77.6 \%)$ & $85(86.7 \%)$ & 0.121 & \\
\hline & Yes & $26(22.4 \%)$ & 13(13.3\%) & & \\
\hline \multirow[t]{2}{*}{ Antireflux treatment (\%) } & No & $82(82.0 \%)$ & $60(75.9 \%)$ & 0.420 & \\
\hline & Yes & 18(18.0\%) & $19(24.1 \%)$ & & \\
\hline \multirow[t]{2}{*}{ Barretts esophagus (\%) } & No & $116(92.1 \%)$ & $77(93.9 \%)$ & 0.821 & \\
\hline & Yes & $10(7.9 \%)$ & $5(6.1 \%)$ & & \\
\hline \multirow[t]{2}{*}{ TP53 status (\%) } & Mut & $99(52.7 \%)$ & 73(39.7\%) & 0.013 & exact \\
\hline & WT & $89(47.3 \%)$ & $111(60.3 \%)$ & & \\
\hline \multirow[t]{2}{*}{ PIK3CA status (\%) } & Mut & $25(13.3 \%)$ & $34(18.5 \%)$ & 0.202 & exact \\
\hline & WT & $163(86.7 \%)$ & $150(81.5 \%)$ & & \\
\hline Age [median (IQR)] & & $68.00[58.00,74.00]$ & $67.00[58.00,72.00]$ & 0.367 & nonnorm \\
\hline
\end{tabular}

effect on pancreatic cancer cells (24). The expression of HTRA3 in lung cancer tissue was significantly down-regulated. HTRA3 promoted drug-induced apoptosis of lung cancer cells (11). The expression of HTRA3 was negatively correlated with lymph node metastasis in breast cancer, but not with positive or negative expression of ER and PR (12). In non-small cell lung cancer cell lines, exogenous transforming growth factor $\beta-1$ significantly reduced HTRA3 expression during EMT (10).

However, HTRA 3 can promote tumor progression in other malignancies. recent studies have also shown that HTRA3 was highly expressed in thyroid carcinoma and related to the occurrence of thyroid cancer (25). HTRA3 was related to the 
TABLE 2 | HTRA3 expression association with clinical pathological characteristics (logistic regression).

Characteristics

T stage (T3\&T4 vs. T1\&T2)

$\mathrm{N}$ stage (N1\&N2\&N3 vs. N0)

$\mathrm{M}$ stage (M1 vs. M0)

Pathologic stage (Stage III \& Stage IV vs. Stage I \& Stage II)

Histological type (Diffuse Type vs. Tubular Type)

Histologic grade (G3 vs. G1\&G2)

Primary therapy outcome (CR vs. PD\&SD\&PR)

Tumor status (With tumor vs. Tumor free)

Residual tumor (R1\&R2 vs. R0)

TP53 status (Mut vs. WT)

PIK3CA status (Mut vs. WT)
Odds Ratio in HTRA3 expression

367
357
355
352
132
366
317
337
329
372
372

Odds Ratio (OR)

$P$ value

$2.39(1.49-3.90)$

$0.88(0.56-1.39)$

$0.95(0.41-2.15)$

1.12(0.74-1.70)

$2.34(1.17-4.78)$

2.14(1.40-3.29)

$1.56(0.95-2.58)$

$1.11(0.71-1.72)$

$0.80(0.38-1.68)$

$0.59(0.39-0.89)$

$1.48(0.85-2.61)$
$<0.001$

0.592

0.893

0.598

0.017

$<0.001$

0.080

0.659

0.560

0.012

0.173
A

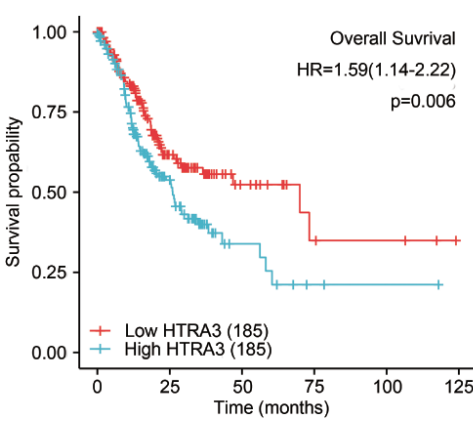

D

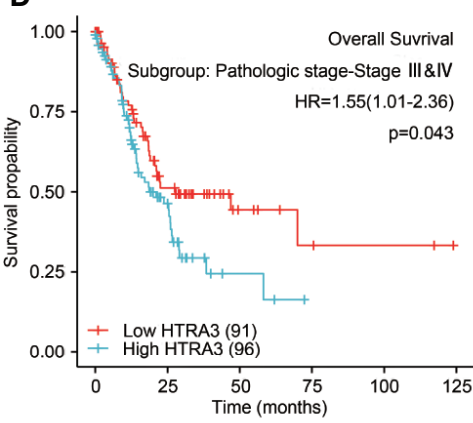

G

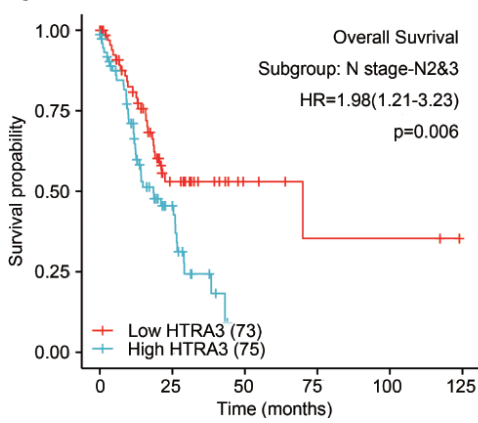

B

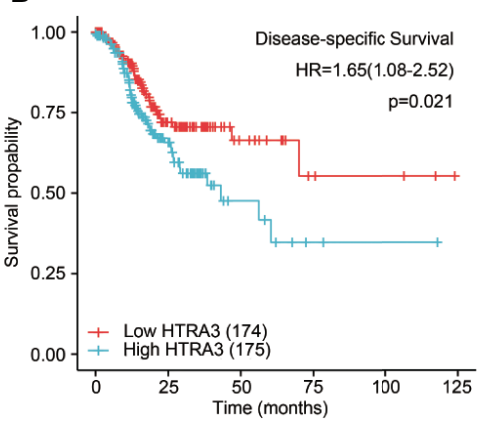

C

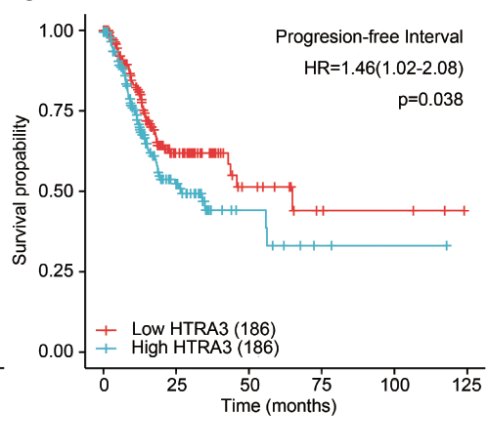

$\mathbf{F}$

E
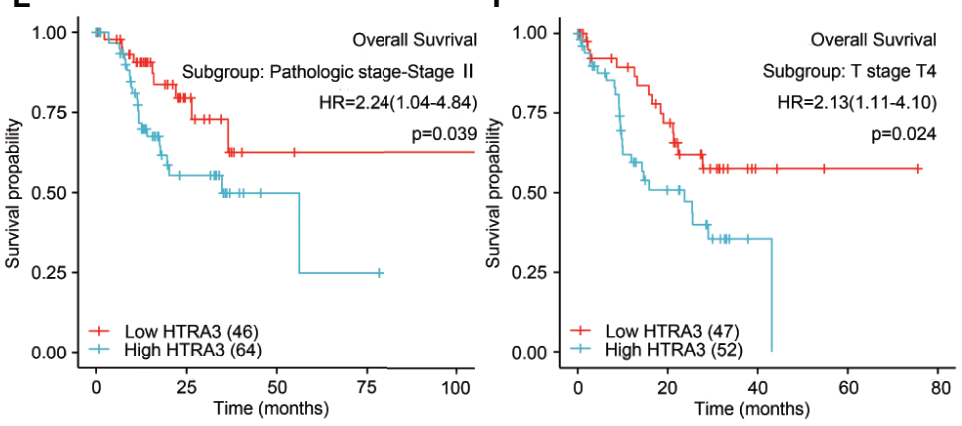

I

H

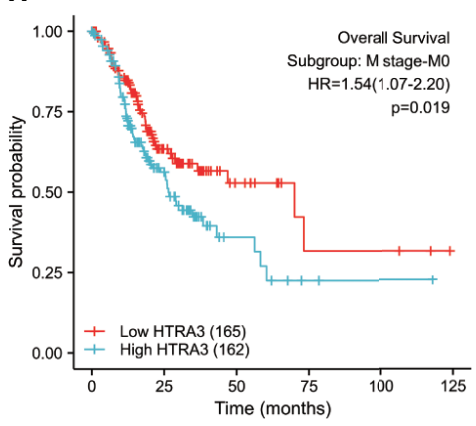

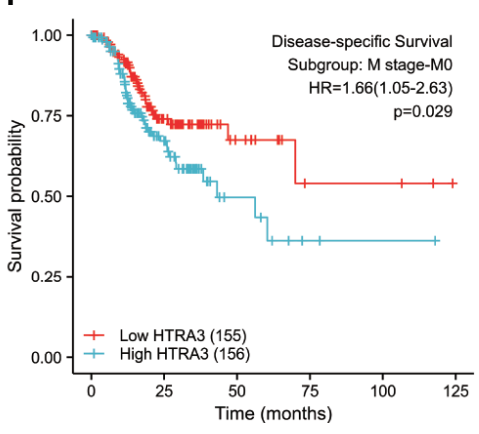

FIGURE 5 | Kaplan-Meier survival curves comparing the high and low expression of HTRA3 in GC. (A-C) Survival curves of OS, DSS, and PFI between HTRA3-high and -low patients with GC. (D-H) OS survival curves of stage III \& IV, stage III, T4, N2\&3, and MO subgroups between HTRA3-high and -low patients with GC. (I) DSS survival curves of MO subgroup between HTRA3-high and -low patients with GC. GC, gastric cancer; OS, overall survival; DSS, disease specific survival; PFI, progression free interval. 
TABLE 3 | Univariate regression and multivariate survival method (Overall Survival) of prognostic covariates in patients with gastric cancer.

\begin{tabular}{|c|c|c|c|c|c|}
\hline Characteristics & $\begin{array}{c}\text { Total } \\
(\mathrm{N})\end{array}$ & $\begin{array}{l}\text { HR (95\% Cl) Univariate } \\
\text { analysis }\end{array}$ & $\begin{array}{l}\text { P value Univariate } \\
\text { analysis }\end{array}$ & $\begin{array}{l}\text { HR }(95 \% \mathrm{Cl}) \text { Multivariate } \\
\text { analysis }\end{array}$ & $\begin{array}{l}\text { P value Multivariate } \\
\text { analysis }\end{array}$ \\
\hline T stage (T3\&T4 vs. T1\&T2) & 362 & $1.719(1.131-2.612)$ & 0.011 & $1.103(0.582-2.092)$ & 0.763 \\
\hline N stage (N1\&N2\&N3 vs. N0) & 352 & $1.925(1.264-2.931)$ & 0.002 & $2.394(1.051-5.452)$ & 0.038 \\
\hline M stage (M1 vs. M0) & 352 & $2.254(1.295-3.924)$ & 0.004 & $0.677(0.263-1.744)$ & 0.419 \\
\hline $\begin{array}{l}\text { Pathologic stage (Stage III \& Stage IV vs. Stage I } \\
\text { \& Stage II) }\end{array}$ & 347 & $1.947(1.358-2.793)$ & $<0.001$ & $0.852(0.423-1.717)$ & 0.655 \\
\hline Histologic grade (G3 vs. G1\&G2) & 361 & $1.353(0.957-1.914)$ & 0.087 & $1.273(0.783-2.071)$ & 0.330 \\
\hline Histological type (Diffuse Type vs. Tubular Type) & 132 & $1.077(0.620-1.872)$ & 0.793 & & \\
\hline Primary therapy outcome (CR vs. PD\&SD\&PR) & 313 & $0.237(0.163-0.344)$ & $<0.001$ & $0.426(0.241-0.755)$ & 0.003 \\
\hline Residual tumor (R1\&R2 vs. R0) & 325 & $3.445(2.160-5.494)$ & $<0.001$ & $1.503(0.760-2.971)$ & 0.241 \\
\hline Age (>65 vs. $<=65)$ & 367 & $1.620(1.154-2.276)$ & 0.005 & $1.921(1.215-3.036)$ & 0.005 \\
\hline $\begin{array}{l}\text { Race (Asian \& Black or African American vs. } \\
\text { White) }\end{array}$ & 320 & $0.801(0.515-1.247)$ & 0.326 & & \\
\hline Gender (Male vs. Female) & 370 & $1.267(0.891-1.804)$ & 0.188 & & \\
\hline $\begin{array}{l}\text { Anatomic neoplasm subdivision (Fundus/Body } \\
\text { vs. Antrum/Distal) }\end{array}$ & 267 & $0.965(0.651-1.430)$ & 0.858 & & \\
\hline Reflux history (Yes vs. No) & 213 & $0.582(0.291-1.162)$ & 0.125 & & \\
\hline Antireflux treatment (Yes vs. No) & 179 & $0.756(0.422-1.353)$ & 0.346 & & \\
\hline Barretts esophagus (Yes vs. No) & 207 & $0.892(0.326-2.441)$ & 0.824 & & \\
\hline TP53 status (Mut vs. WT) & 367 & $0.865(0.621-1.205)$ & 0.392 & & \\
\hline PIK3CA status (Mut vs. WT) & 367 & $0.623(0.370-1.048)$ & 0.075 & $0.468(0.248-0.881)$ & 0.019 \\
\hline Tumor status (With tumor vs. Tumor free) & 333 & $5.420(3.640-8.071)$ & $<0.001$ & $3.526(1.981-6.276)$ & $<0.001$ \\
\hline HTRA3 (High vs. Low) & 370 & $1.590(1.140-2.219)$ & 0.006 & $2.315(1.447-3.703)$ & $<0.001$ \\
\hline
\end{tabular}

TABLE 4 | The prognostic value of HTRA3 (Overall Survival) in various gastric cancer subgroups.

\begin{tabular}{lccc}
\hline Characteristics & N (\%) & HR (95\% Cl) & P value \\
\hline T stage & & & \\
T1\&T2 & $96(27)$ & $1.406(0.667-2.963)$ & 0.370 \\
T3 & $167(46)$ & $1.254(0.780-2.016)$ & 0.349 \\
T4 & $99(27)$ & $2.130(1.106-4.104)$ & 0.024 \\
N stage & & & \\
N0 & $107(30)$ & $1.460(0.666-3.198)$ & 0.344 \\
N1 & $97(28)$ & $1.362(0.736-2.521)$ & 0.325 \\
N2\&N3 & $148(42)$ & $1.979(1.214-3.227)$ & 0.006 \\
M stage & & & \\
M0 & $327(93)$ & $1.536(1.075-2.196)$ & 0.019 \\
M1 & $25(7)$ & $1.551(0.527-4.564)$ & 0.425 \\
Pathologic stage & $50(14)$ & $0.977(0.281-3.394)$ & 0.971 \\
Stage I & $110(32)$ & $2.244(1.040-4.838)$ & 0.039 \\
Stage II & $187(54)$ & $1.546(1.013-2.357)$ & 0.043 \\
Stage III \& Stage IV & & & \\
\hline
\end{tabular}

acquisition of invasive phenotype of oral squamous cell carcinoma and may be a potential prognostic indicator of oral cancer (14). HTRA3 is highly expressed in the stroma of the invasive front of colorectal cancer. The high expression of HTRA3 in tumor core was significantly correlated with the decrease of 5-year overall survival rate. In addition, the expression of HTRA3 in the peritumor stroma of patients with stage II colorectal cancer is related to high grade tumorous budding, which may be a new marker of poor prognosis (13).

To further investigate the functions of HTRA3 in GC, we performed GO, GESA, and ssGSEA analyses using TCGA data. The results revealed that NF- $\kappa \mathrm{B}$ signaling pathway, YAP1/ WWTR1/TAZ pathway, TGF $\beta$ signaling pathway, RAS/CA2P/ NMDA pathway, RHO/CIT pathway, and RAC1 signaling pathway were differentially enriched in the HTRA3-high phenotype and fewer abundance of adaptive immune cells were also observed. These data suggested that HTRA3 might serve as a potential prognostic marker and therapeutic target in GC.

HTRA3 might promote the instability of cytoskeleton, thereby regulating the EMT process of various tumor cells. And HTRA3 is a potential therapeutic target involved in various cancers (21). Researchers revealed that the modulation of HTRA3 in tumorigenesis might be dual, either inhibitory or promoting, depending on the specific tissues, stages of cancer progression etc. On one hand, HTRA3 expression is low in endometrial cancer, ovarian cancer, and lung cancer $(23,26)$. HTRA3 promotes druginduced apoptosis through XIAP cleavage in lung cancer cells (11). In non-small cell lung cancer (NSCLS) cell lines, overexpression of HTRA3 inhibits the carcinogenesis of TGF- $\beta 1$, thus inhibiting tumor metastasis in the early stage of cancer. The role of HTRA3 is weakened, and transforming growth factor $\beta 1$ effectively promoted EMT without HTRA3 brakes in the later stage of cancer (10). The ectopic expression of HTRA3 in pancreatic cancer leads to the decrease of cell proliferation and the increase of the expression of apoptotic protein Bax, suggesting that HTRA3 has anti-tumor effect on pancreatic cancer cells (24). On the other hand, HTRA3 is highly expressed in oral squamous cell carcinoma, thyroid cancer and colorectal cancer, and the acquisition of invasive phenotype in oral squamous cell carcinoma and colorectal tumor is closely related to poor prognosis $(13,14,25)$. Here, we found that HTRA3 is highly expressed in GC. The overexpression of HTRA3 was significantly correlated with histological type, histological grade, clinical stage, T stage, and TP53 status of gastric cancer. HTRA3-high patients with GC had worse histological types (diffuse type), lower tumor differentiation, later clinicopathological stages (especially greater primary tumor growth), and were less prone to have TP53 

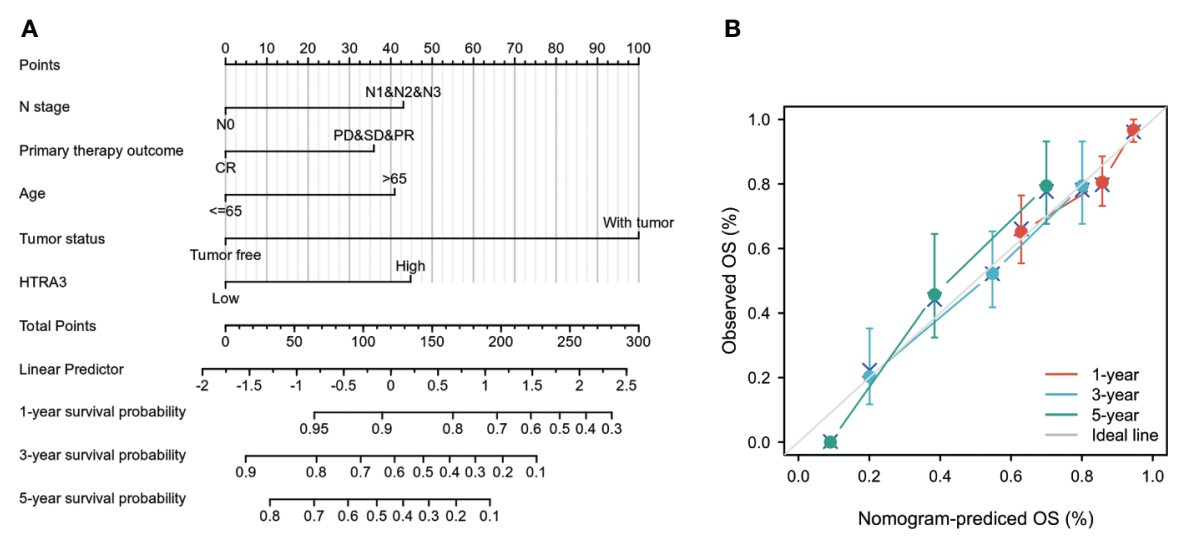

FIGURE 6 | A quantitative method to predict GC patients' probability of 1-, 3-, and 5-year OS. (A) A nomogram for predicting the probability of 1-, 3-, and 5- year OS for GC patients. (B) Calibration plots of the nomogram for predicting the probability of OS at 1, 3, and 5 years. GC, gastric cancer; OS, overall survival.

mutations, highlighting the potential role of HTRA3 in the development of GC.

Given the limited data on HTRA3 function, we performed functional annotation based on GO and GESA. We demonstrated that HTRA3-high phenotype was associated with NF- $\kappa \mathrm{B}$ signaling pathway, YAP1/WWTR1/TAZ signaling pathway, TGF $\beta$ pathway, RAS/CA2P/NMDA pathway, RHO/ CIT pathway, and RAC1 signaling pathway. Recent studies have elucidated that Enhanced activity of NF- $\mathrm{KB}$ signaling pathway can promote proliferation, metastasis, and angiogenesis of GC cells (27). YAP1/TAZ activity controls GC stem cells tumorigenic properties (28), indicating that HTRA3 may promote GC cell growth, metastasis, and poor survival via the NF- $\kappa B$ and YAP1/WWTR1/TAZ pathway. Additionally, the PPI network of HTRA3-associated genes was constructed in this study, and these genes were involved in various signaling pathways and biological processes. In future studies, we will further determine the correlation between HTRA3-associated genes and the prognosis of GC.

Stromal cells in tumor microenvironment can change the carcinogenic characteristics of tumor cells. Among them, tumorinfiltrating lymphocytes (TILs) play an important role in the occurrence and development of tumors $(29,30)$. TILs establish a complex intercellular interaction network, which helps improve and maintain the immunosuppressive microenvironment, promote immune escape, and thus promote tumor progression (31). Our results demonstrated there were more NK cells, TAMs, and fewer Th17 cells in the HTRA3-high GC group than those in the HTRA3-low GC group, suggesting the improvement of innate immunity was accompanied by the decrease of adaptive immunity. Furthermore, infiltrating NK cells and TAMs in the tumor microenvironment have strong immunosuppressive activity, which reduces the secretion of IFN- $\gamma$ and induces T cell dysfunction $(32,33)$. Additionally, the subgroup with more Th17 cell infiltration in breast and ovarian cancer is less likely to have lymph node metastasis and more likely to have a better prognosis, which suggests that Th17 cells have anti-tumor effect (34-36). Therefore, our data elucidated the immunosuppression induced by fewer Th17 cells in the primary tumor microenvironment might result in a lower 10-year survival rate in HTRA3-high patients with GC.

High level of HTRA3 expression was correlated with poor prognosis of GC in stage III - IV, T4, N2-3, and M0 subgroups, with the highest HR for poor OS, DSS, and PFI when HTRA3 was highly expressed in GC. We found that the expression of HTRA3 remained a powerful predictor of prognosis within these subsets, suggesting that HTRA3 was independent of these important clinicopathological parameters. Subsequently, a nomogram with comprehensive evaluation combining HTRA3 with other important clinical patterns (HTRA3 status, N stage, primary therapy outcome, age status, and tumor status) was performed. Based on the calibration plot, there was a favorable consistency between the actual and predicted values for 1-, 3-, 5-year OS. Our model was constructed based on the complementary perspective for respective tumors and provided a personalized score for individual patients. Consequently, our nomogram could be a valuable new prognostic method for clinicians in the future.

Although these results improved our understanding of the relationship between HTRA3 and GC, there were some limitations. First, to clarify the specific role of HTRA3 in the development of GC comprehensively, several clinical factors and parameters should be considered, such as the details on treatments received by patients involved. However, this information was lacked or inconsistent in public databases because the experiments were performed in different centers. Second, the number of healthy subjects used as controls was considerably different from that of patients with cancer in the current study, hence additional studies were required to maintain a balance of sample size. Third, although multicenter study in public databases intends to complement the drawbacks of single center study, retrospective studies have their limitations, especially non-uniform intervening measures, and lacking of some information. Therefore, a prospective study should be performed in the future to avoid analysis bias arising due to the retrospective nature of the current study. Lastly, since the current study was performed based on RNA sequencing from 
TCGA database only, it is necessary to further study the direct mechanism of HTRA3 in GC.

In this study, we firstly reported that the high expression of HTRA3 was significantly associated with the progression, poor survival, and immune infiltration of GC, which might promote tumorigenesis through abnormal inflammation and immune response. HTRA3 has the potential to predict treatment outcomes and may become a new biomarker of GC. The mechanism of HTRA3 promoting the progression and metastasis of GC will be verified in further studies. This study provided a new and promising insight for further elucidating the clinicopathological significance and molecular pathogenesis of GC.

\section{DATA AVAILABILITY STATEMENT}

The original contributions presented in the study are included in the article/Supplementary Material. Further inquiries can be directed to the corresponding author.

\section{ETHICS STATEMENT}

Ethical review and approval were not required for the study on human participants in accordance with the local legislation and institutional requirements. The patients/participants provided their written informed consent to participate in this study.

\section{AUTHOR CONTRIBUTIONS}

B-CJ contributed to concept and design of this article. CJ participated in manuscript writing. L-SS, FX, and NN participated in data collection and data analysis. H-LG, J-WD, and NZ analyzed and interpreted data. All authors contributed to the article and approved the submitted version.

\section{FUNDING}

This work was supported by the Medical and Health Science Technology Planning Projects of Liaoning Province of China

\section{REFERENCES}

1. Siegel RL, Miller KD, Jemal A. Cancer statistics, 2020. CA: Cancer J Clin (2020) 70(1):7-30. doi: 10.3322/caac.21590

2. Miller KD, Nogueira L, Mariotto AB, Rowland JH, Yabroff KR, Alfano CM, et al. Cancer treatment and survivorship statistics, 2019. CA: Cancer J Clin (2019) 69(5):363-85. doi: 10.3322/caac.21565

3. Yan HHN, Siu HC, Law S, Ho SL, Yue SSK, Tsui WY, et al. A Comprehensive Human Gastric Cancer Organoid Biobank Captures Tumor Subtype Heterogeneity and Enables Therapeutic Screening. Cell Stem Cell (2018) 23 (6):882-97.e11. doi: 10.1016/j.stem.2018.09.016

4. Pietrantonio F, Miceli R, Raimondi A, Kim YW, Kang WK, Langley RE, et al. Individual Patient Data Meta-Analysis of the Value of Microsatellite
(20170540986 and 2019-ZD-0739), the Science and Technology Planning Project of Shenyang City (18-014-4-22) and 50 Talent Project of Shengjing Hospital of China Medical University.

\section{SUPPLEMENTARY MATERIAL}

The Supplementary Material for this article can be found online at: https://www.frontiersin.org/articles/10.3389/fonc.2020. 603480/full\#supplementary-material

SUPPLEMENTAL FIGURE 1 | Experimental verification of differential mRNA and protein expression of HTRA3 in GC samples and cell lines. (A) HTRA3 mRNA and protein expression in 44 paired adjacent noncancerous tissues and GC tissues. (B) HTRA3 mRNA and protein expression in GC cell lines (SGC7901, MKN45, MGC803, BGC823, and HGC27) and a gastric epithelial cell line (GES1). GAPDH was used as a loading control. Results are representative of three different experiments and data are expressed as mean \pm standard deviation. HTRA3, HtrA serine peptidase 3; GC, gastric cancer.

SUPPLEMENTAL FIGURE 2 | The protein to protein interactions network constructed by protein to protein interactions pairs based on the STRING database.

SUPPLEMENTAL TABLE 1 | Clinical characteristics of gastric cancer patients based on TCGA.

SUPPLEMENTAL TABLE 2 | 50 items of HTRA3-related differential expressed Genes.

SUPPLEMENTAL TABLE 3 | 50 items of Gene Ontology enrichment analysis.

SUPPLEMENTAL TABLE 4 | Gene sets enriched in phenotype high. NES, normalized enrichment score; p. adjusted, adjusted P-value.

SUPPLEMENTARY TABLE 5 | 50 items of protein to protein interactions of HTRA3.

SUPPLEMENTARY TABLE 6 | Univariate regression and multivariate survival method (Disease Specific Survival) of prognostic covariates in patients with gastric cancer.

SUPPLEMENTARY TABLE 7 | Univariate regression and multivariate survival method (Progression Free Interval) of prognostic covariates in patients with gastric cancer.

SUPPLEMENTARY TABLE 8 | The prognostic value of HTRA3 (Disease Specific Survival) in various gastric cancer subgroups.

SUPPLEMENTARY TABLE 9 | The prognostic value of HTRA3 (Progression Free Interval) in various gastric cancer subgroups.

Instability As a Biomarker in Gastric Cancer. J Clin Oncol Off J Am Soc Clin Oncol (2019) 37(35):3392-400. doi: 10.1200/jco.19.01124

5. Lordick F. Chemotherapy for resectable microsatellite instability-high gastric cancer? Lancet Oncol (2020) 21(2):203. doi: 10.1016/s1470-2045(20)30012-7

6. Xu B, Huang C, Yang X, Li X, Li L, Ding Y. Significance and prognostic role of human epidermal growth factor receptor 2 and RAB1A expression in gastric cancer. Oncol Lett (2018) 15(4):5185-92. doi: 10.3892/ol.2018.7992

7. Shitara K, Iwata H, Takahashi S, Tamura K, Park H, Modi S, et al. Trastuzumab deruxtecan (DS-8201a) in patients with advanced HER2positive gastric cancer: a dose-expansion, phase 1 study. Lancet Oncol (2019) 20(6):827-36. doi: 10.1016/s1470-2045(19)30088-9

8. Nie GY, Hampton A, Li Y, Findlay JK, Salamonsen LA. Identification and cloning of two isoforms of human high-temperature requirement factor A3 (HtrA3), characterization of its genomic structure and comparison of its 
tissue distribution with HtrA1 and HtrA2. Biochem J (2003) 371:39-48. doi: $10.1042 / b j 20021569$

9. Acharya S, Dutta S, Bose K. A distinct concerted mechanism of structural dynamism defines activity of human serine protease HtrA3. Biochem $J(2020)$ 477(2):407-29. doi: 10.1042/bcj20190706

10. Zhao J, Feng M, Liu D, Liu H, Shi M, Zhang J, et al. Antagonism between HTRA3 and TGF $\beta 1$ Contributes to Metastasis in Non-Small Cell Lung Cancer. Cancer Res (2019) 79(11):2853-64. doi: 10.1158/0008-5472.can-18-2507

11. Wenta T, Rychlowski M, Jurewicz E, Jarzab M, Zurawa-Janicka D, Filipek A, et al. The HtrA3 protease promotes drug-induced death of lung cancer cells by cleavage of the X-linked inhibitor of apoptosis protein (XIAP). FEBS J (2019) 286(22):4579-96. doi: 10.1111/febs.14977

12. Yin $\mathrm{Y}, \mathrm{Wu} \mathrm{M}, \mathrm{Nie} \mathrm{G}$, Wang $\mathrm{K}$, Wei J, Zhao $\mathrm{M}$, et al. HtrA3 is negatively correlated with lymph node metastasis in invasive ductal breast cancer. Tumour Biol J Int Soc Oncodevelopm Biol Med (2013) 34(6):3611-7. doi: 10.1007/s13277-013-0942-5

13. Forse CL, Rahimi M, Diamandis EP, Assarzadegan N, Dawson H, Grin A, et al. HtrA3 stromal expression is correlated with tumor budding in stage II colorectal cancer. Exp Mol Pathol (2017) 103(1):94-100. doi: 10.1016/ j.yexmp.2017.07.002

14. Moriya Y, Uzawa N, Morita T, Mogushi K, Miyaguchi K, Takahashi K, et al. The high-temperature requirement factor $\mathrm{A} 3$ ( $\mathrm{HtrA} 3)$ is associated with acquisition of the invasive phenotype in oral squamous cell carcinoma cells. Oral Oncol (2015) 51(1):84-9. doi: 10.1016/j.oraloncology.2014.10.001

15. Love MI, Huber W, Anders S. Moderated estimation of fold change and dispersion for RNA-seq data with DESeq2. Genome Biol (2014) 15(12):550. doi: 10.1186/s13059-014-0550-8

16. Zhou Y, Zhou B, Pache L, Chang M, Khodabakhshi AH, Tanaseichuk O, et al. Metascape provides a biologist-oriented resource for the analysis of systems-level datasets. Nat Commun (2019) 10(1):1523. doi: 10.1038/s41467-019-09234-6

17. Yu G, Wang LG, Han Y, He QY. clusterProfiler: an R package for comparing biological themes among gene clusters. Omics J Integr Biol (2012) 16(5):284-7. doi: 10.1089/omi.2011.0118

18. Szklarczyk D, Gable AL, Lyon D, Junge A, Wyder S, Huerta-Cepas J, et al. STRING v11: protein-protein association networks with increased coverage, supporting functional discovery in genome-wide experimental datasets. Nucleic Acids Res (2019) 47(D1):D607-13. doi: 10.1093/nar/gky1131

19. Bindea G, Mlecnik B, Tosolini M, Kirilovsky A, Waldner M, Obenauf AC, et al. Spatiotemporal dynamics of intratumoral immune cells reveal the immune landscape in human cancer. Immunity (2013) 39(4):782-95. doi: 10.1016/j.immuni.2013.10.003

20. Theleyre S, Mottier S, Masson D, Denis MG. HtrA3 is regulated by 15 -deoxyDelta12,14-prostaglandin J2 independently of PPARgamma in clear cell renal cell carcinomas. Biochem Biophys Res Commun (2010) 394(3):453-8. doi: 10.1016/j.bbrc.2009.11.163

21. Wenta T, Zurawa-Janicka D, Rychlowski M, Jarzab M, Glaza P, Lipinska A, et al. HtrA3 is a cellular partner of cytoskeleton proteins and TCP1 $\alpha$ chaperonin. J Proteomics (2018) 177:88-111. doi: 10.1016/j.jprot.2018.02.022

22. Narkiewicz J, Lapinska-Szumczyk S, Zurawa-Janicka D, Skorko-Glonek J, Emerich J, Lipinska B. Expression of human HtrA1, HtrA2, HtrA3 and TGFbetal genes in primary endometrial cancer. Oncol Rep (2009) 21(6):1529-37. doi: $10.3892 /$ or_00000385

23. Zhao M, Ding JX, Nie GY, Wei J, Li Y, Yin XY, et al. HTRA3 is reduced in ovarian cancers regardless of stage. Cancer Invest (2014) 32(9):464-9. doi: $10.3109 / 07357907.2014 .958496$
24. Li Y, Gong L, Qi R, Sun Q, Xia X, He H, et al. Paeoniflorin suppresses pancreatic cancer cell growth by upregulating HTRA3 expression. Drug Design Dev Ther (2017) 11:2481-91. doi: 10.2147/dddt.s134518

25. Zurawa-Janicka D, Kobiela J, Galczynska N, Stefaniak T, Lipinska B, Lachinski A, et al. Changes in expression of human serine protease HtrAl, HtrA2 and HtrA3 genes in benign and malignant thyroid tumors. Oncol Rep (2012) 28 (5):1838-44. doi: 10.3892/or.2012.1988

26. Lv Q, Yang B, Ning C, Xie B, Nie G, Chen X, et al. Hypoxia is involved in the reduction of HtrA3 in patients with endometrial hyperplasia and cancer Biochem Biophys Res Commun (2018) 503(4):2918-23. doi: 10.1016/ j.bbrc.2018.08.070

27. Chen P, Guo H, Wu X, Li J, Duan X, Ba Q, et al. Epigenetic silencing of microRNA-204 by Helicobacter pylori augments the NF- $\mathrm{KB}$ signaling pathway in gastric cancer development and progression. Carcinogenesis (2019) 41(4):430-41. doi: 10.1093/carcin/bgz143

28. Giraud J, Molina-Castro S, Seeneevassen L, Sifré E, Izotte J, Tiffon C, et al. Verteporfin targeting YAP1/TAZ-TEAD transcriptional activity inhibits the tumorigenic properties of gastric cancer stem cells. Int J Cancer (2020) 146 (8):2255-67. doi: 10.1002/ijc.32667

29. Demaria O, Vivier E. Immuno-Oncology beyond TILs: Unleashing TILCs. Cancer Cell (2020) 37(4):428-30. doi: 10.1016/j.ccell.2020.03.021

30. Callahan MK, Wolchok JD. Recruit or Reboot? How Does Anti-PD-1 Therapy Change Tumor-Infiltrating Lymphocytes? Cancer Cell (2019) 36(3):215-7. doi: 10.1016/j.ccell.2019.08.009

31. Yu YR, Imrichova H, Wang H, Chao T, Xiao Z, Gao M, et al. Disturbed mitochondrial dynamics in CD8(+) TILs reinforce T cell exhaustion. Nat Immunol (2020) 21(12):1540-51. doi: 10.1038/s41

32. Platonova S, Cherfils-Vicini J, Damotte D, Crozet L, Vieillard V, Validire P, et al. Profound coordinated alterations of intratumoral NK cell phenotype and function in lung carcinoma. Cancer Res (2011) 71(16):5412-22. doi: 10.1158/ 0008-5472.can-10-4179

33. Solinas G, Germano G, Mantovani A, Allavena P. Tumor-associated macrophages (TAM) as major players of the cancer-related inflammation. J Leukocyte Biol (2009) 86(5):1065-73. doi: 10.1189/jlb.0609385

34. Yang L, Qi Y, Hu J, Tang L, Zhao S, Shan B. Expression of Th17 cells in breast cancer tissue and its association with clinical parameters. Cell Biochem Biophysics (2012) 62(1):153-9. doi: 10.1007/s12013-011-9276-3

35. Salazar Y, Zheng X, Brunn D, Raifer H, Picard F, Zhang Y, et al. Microenvironmental Th9- and Th17- lymphocytes induce metastatic spreading in lung cancer. J Clin Invest (2020) 130(7):3560-75. doi: 10.1172/ jci124037

36. Punkenburg E, Vogler T, Büttner M, Amann K, Waldner M, Atreya R, et al. Batf-dependent Th17 cells critically regulate IL-23 driven colitis-associated colon cancer. Gut (2016) 65(7):1139-50. doi: 10.1136/gutjnl-2014-308227

Conflict of Interest: The authors declare that the research was conducted in the absence of any commercial or financial relationships that could be construed as a potential conflict of interest.

Copyright (๑) $2020 \mathrm{Ji}$, Sun, Xing, Niu, Gao, Dai, Zhou and Jiang. This is an open-access article distributed under the terms of the Creative Commons Attribution License (CC BY). The use, distribution or reproduction in other forums is permitted, provided the original author(s) and the copyright owner(s) are credited and that the original publication in this journal is cited, in accordance with accepted academic practice. No use, distribution or reproduction is permitted which does not comply with these terms. 